\title{
Delayed Primary Closure of the Septic Open Abdomen with a Dynamic Closure System
}

\author{
Froukje J. Verdam • Dennis E. J. G. J. Dolmans • \\ Maarten J. Loos • Menno H. Raber • Ralph J. de Wit • \\ Jan A. Charbon · Jos P. A. M. Vroemen
}

Published online: 18 August 2011

(c) The Author(s) 2011. This article is published with open access at Springerlink.com

\begin{abstract}
Background The major challenge in the management of patients with an infected open abdomen (OA) is to control septic peritonitis and intra-abdominal fluid secretion, and to facilitate repeated abdominal exploration, while preserving the fascia for delayed primary closure. We here present a novel method for closure of the infected OA, based on continuous dynamic tension, in order to achieve re-approximation of the fascial edges of the abdominal wall.

Methods Eighteen cases with severe peritonitis of various origin (e.g., gastrointestinal perforations, anastomotic leakage) were primarily stabilized by laparostomy, sealed with
\end{abstract}

Electronic supplementary material The online version of this article (doi:10.1007/s00268-011-1210-8) contains supplementary material, which is available to authorized users.

F. J. Verdam ( $\square)$ · D. E. J. G. J. Dolmans · J. P. A. M. Vroemen Department of General Surgery, Amphia Hospital,

P.O. Box 90158, 4800 RK Breda, The Netherlands

e-mail: froukjeverdam@yahoo.com

J. P. A. M. Vroemen

e-mail: jvroemen@amphia.nl

F. J. Verdam

Department of General Surgery, Maastricht University Medical

Centre, Maastricht, The Netherlands

D. E. J. G. J. Dolmans

Department of General Surgery, Atrium Medical Center,

Heerlen, The Netherlands

M. J. Loos · J. A. Charbon

Department of General Surgery, Màxima Medical Centre,

Veldhoven, The Netherlands

M. H. Raber - R. J. de Wit

Department of General Surgery, Medical Spectrum Twente,

Enschede, The Netherlands either the vacuum-assisted closure abdominal dressing or the Bogotá bag. After hemodynamic stabilization and control of the sepsis, the Abdominal Re-approximation Anchor System (ABRA; Canica Design, Almonte, Ontario, Canada) was applied. This system approximates the wound margins through dynamic traction exerted by transfascial elastomers. Before ABRA application, 5/18 patients had a grade 2B, 2/18 a grade 3 , and $11 / 18$ a grade or 4 status according to the open abdomen classification of Björck.

Results In this severely ill population the mean time before ABRA system application was 12 days (range: 2-39 days). Two of 18 patients died of non-ABRA-related causes within three weeks. In 14 of the remaining 16 patients (88\%) primary abdominal closure of the midline was accomplished in 15 days (range: 7-30 days). The other two patients needed a component separation technique according to Ramirez to reach closure. However, secondary wound dehiscence occurred in both these patients. Two thirds of patients (12/ 18) developed pressure sores to the skin and/or dermis, but all healed without further complications. During outpatient clinic follow-up, 4/14 successfully closed patients still developed a midline hernia.

Conclusions Delayed primary closure of OA in septic patients could be achieved in $88 \%$ with this new approximation system. However, the risk of hernia development remained. We consider this system a useful tool in the treatment of septic patients with an open abdomen.

\section{Introduction}

The treatment of the open abdomen (OA) is performed by surgeons of various subspecialties, for example in abdominal compartment syndrome (ACS) after trauma or ruptured aneurysm, or in the case of severe generalized 
peritonitis [1, 2]. All patients with an OA are critically ill and have a high risk of developing major complications, such as multiple organ dysfunction syndrome (30\%-40\%) [3], enterocutaneous fistula $(2 \%-25 \%)$ [4, 5], intraabdominal abscess (83\%), and abdominal wall hernia (around 25\%) [6]. Mortality rates up to 50\% are reported $[1,7]$, and the risk of mortality is even higher in the infected than in the traumatic OA $[1,3,8]$.

The septic patient with an open abdomen first needs to be stabilized. The priorities are to control sepsis, treat the intra-abdominal infection, and prevent multiple organ failure. Secondly, the management of this type of patient is aimed at closure of the abdomen. Many current management modalities used in the infected OA are not primarily intended to close the abdomen (such as draping and suction, wound manager). Those modalities that are aimed at closure (e.g., Wittmann patch, meshes) produce more satisfying success rates [7], but abdominal closure is still not always accomplished. As a consequence, many OA patients often develop large and debilitating hernias of the abdominal wall that require complex repair surgery at a later stage [6].

The Abdominal Re-approximation Anchor system (ABRA, Canica, Almonte, Ontario, Canada) is a novel technique based on dynamic elastic closure. It was designed specifically for the delayed closure of the OA. This closure technique has been used previously in OA of mainly nonseptic origin $[9,10]$. In the present study, we describe the use of this abdominal re-approximation technique in an OA patient cohort with generalized sepsis due to peritonitis.

\section{Materials and methods}

\section{Population characteristics}

We present our experience with the ABRA system in 18 septic cases with OA treatment in three large, nonacademic hospitals in the Netherlands between January 2006 and May 2010. In all centers, one surgeon applied the system and supervised all patients.

The causes for the need of OA treatment in this septic population varied; spontaneous perforations occurred in 8 patients: stomach $(n=1)$, coecum $(n=4)$, and colon $(n=3)$. These spontaneous perforations occurred due to either an inflammatory process $(n=6)$ or due to an underlying malignancy $(n=2)$. Anastomotic leakage was found in 7 cases; after diverticulitis $(n=3)$ or malignancy $(n=4)$. Three patients suffered from a traumatic bowel perforation; one patient from a small bowel perforation during hernia repair surgery after Dukes $\mathrm{C}$ colon carcinoma, one after restoration of intestinal continuity after
Hartmann procedure, and one patient suffered a selfinflicted perforation of the rectum, duodenum, and right hemidiaphragm by a foreign body.

Before any kind of abdominal wall approximation method could be applied, our main concern was to reach hemodynamic stability in these septic patients. In the first phase of open abdominal treatment, control of the sepsis was established in the intensive care unit (ICU), by mechanical ventilation, antibiotic treatment, decompression of the abdominal cavity, and repeated lavage. Next to these general supportive measures, the laparostomy was sealed by either the VAC Abdominal Dressing (Kinetic Concepts, Inc. San Antonio, TX; $n=9$ ) or the Bogotá bag $(n=9)$. These methods allow decompression of the abdomen, sequential lavage, and debridement, and they do not damage the midline fascia. VAC therapy has the additional advantage of evacuating the inflammatory exudate. The APACHE II score, the Mannheim peritonitis index [11], and the abdomen classification according to Björck et al. [12] were retrospectively calculated from the patient records and are presented in Table 1.

After stabilization of the abdominal sepsis, patients were considered for delayed primary closure. If it was expected that abdominal closure with conventional methods could not be achieved within a few days, selection for the

Table 1 Patient characteristics

\begin{tabular}{ll}
\hline $\begin{array}{l}\text { Male/female } \\
\text { Mean age, years (range) }\end{array}$ & $14 / 4$ \\
Causes of peritonitis leading to OA & $66(50-90)$ \\
$\quad$ Visceral perforation (inflammatory & \\
$\quad$ origin/malignancy) & $8 / 18(6 / 2)$ \\
Leakage of bowel anastomosis & $7 / 18$ \\
Traumatic bowel perforation & $3 / 18$ \\
Mean APACHE II score (range) & $15(5-29)$ \\
Presence of colostomy & $5 / 18$ \\
Presence of fistula & $3 / 18$ \\
Mean width of the abdominal & $21(17-27)$ \\
defect, cm (range) & \\
OA score according to Björck ${ }^{\mathrm{a}}$ & \\
Grade 2B & $5 / 18$ \\
Grade 3 & $2 / 18$ \\
Grade 4 & $11 / 18$ \\
Mean duration of OA until ABRA & $12(2-39)$ \\
application, days (range) &
\end{tabular}

a The classification for open abdomens according to Björck was noted before the application of the ABRA system: grade 1A, clean OA without adherence between bowel and abdominal wall or fixity of the abdominal wall; grade $1 \mathrm{~B}$, contaminated $\mathrm{OA}$ without adherence/ fixity; grade 2A, clean OA developing adherence/fixity; grade 2B, contaminated OA developing adherence/fixity; grade 3, OA complicated by fistula formation; and grade 4 , frozen OA with adherent/fixed bowel, unable to close surgically, with or without fistula 
application of the ABRA system was based on the clinical judgment of the treating surgeons.

Documentation included the timing of application of the ABRA system, width of the abdominal defect $(\mathrm{cm})$, extent of damage to the fascia (scored as undamaged, damaged, or severely damaged), and the type of abdominal exudate (clear, purulent, or fecal). The presence of a stoma, fistula development, and ABRA-related complications were registered. Moreover, duration of surgery, admission period in the ICU, total hospital admittance, and the occurrence of incisional hernia during outpatient clinic follow-up were recorded. Statistical analysis was performed with SPSS 15.0 (SPSS Inc., Chicago, IL) and Prism 5.0 for Windows (GraphPad Software Inc., San Diego, CA). All data are presented as mean and range.

\section{Technique of ABRA-mediated abdominal closure}

The application of the ABRA system is performed in the operating room. Before application, the wound is surgically debrided, and adhesions to the abdominal contents are carefully dissected to create clean, mobile wound margins (Fig. 1a). Then, a series of midline-crossing elastic bands (elastomers) are surgically inserted through the full thickness of the abdominal wall, in a perpendicular manner at a distance of approximately $5 \mathrm{~cm}$ from the medial fascial margin (Fig. 1b-d). The elastomers are aligned about $3 \mathrm{~cm}$ apart across the defect and fixed to the so-called button anchors at the insertion site (Fig. 1e, f). Before tensioning the elastomers, the viscera protector of the ABRA system (Canica), a perforated silicone sheet, is inserted between the abdominal wall and contents, in order to both protect the viscera and to prevent adhesions during the entire re-approximation process (Fig. 1d).

An adhesive button tail is attached to the anchor to prevent its displacement and tilting. The calibrated elastomers provide continuous dynamic traction in a controlled manner (Fig. 1f). The optimal tension of $80-190 \mathrm{gr} / \mathrm{cm}$ of wound length is obtained by stretching the elastomers 1.5 to twice their tension-free length, which is easily monitored by means of the black-and-white calibration marks (Fig. 1 e and f; Supplementary Fig. 1a) and can be re-adjusted throughout treatment. The remaining defect between the wound margins and the perforated silicone sheet is treated with negative pressure wound therapy (NPWT; VAC system, Kinetic Concepts; Fig. 1g) to ensure drainage of abdominal exudate, and reduce edema [7]. In this way, dynamic traction is evenly distributed, the abdominal exudate is drained by VAC, and the abdomen is progressively closed.

The first few days after application, patients remain in the ICU under general anaesthesia. As soon as the patient's condition permits, treatment can be continued on the surgical ward. With respect to patient bedside care, the buttons and skin are cleansed daily. After this daily routine, the abdominal wall is massaged to reshape the abdomen and to mobilize the abdominal wall over the silicone sheet. This maneuver is performed at bedside according to the ABRA manual and referred to as "the move." In short, both hand palms are placed parallel to the abdominal wound at least $10-15 \mathrm{~cm}$ from the wound edges, and tension-reducing force is applied toward the midpoint (Supplementary Fig. 1b). By this massage, the abdominal oblique muscles are mobilized over the sheet and the abdominal content; advancement of the wound margins toward the midline is facilitated, while the elastomers maintain dynamic apposition of the musculature. The elastomers lose tension and can be re-adjusted after "the move" to the desired tensile strength. Because of the decreasing working tension on the elastomers and ongoing gradual approximation of the wound margins, the tension needs to be checked twice daily and reset if necessary. Every two to three days, the NPWT dressing is changed. If the perforated silicone sheet is visibly soiled with fibrinopurulent discharge, it is taken out, washed in sterile saline, and repositioned. As closure progresses, the sheet often needs to be trimmed. As soon as the fascial edges are re-approximated to $1 \mathrm{~cm}$ distance or less, the midline can be closed. The silicone sheet is removed, and standard fascial closure with non-absorbable sutures is performed in the operating room without the need for additional mesh (Fig. 1h). In almost all cases, the skin can be closed simultaneously. Then the ABRA system can be removed, or it can be maintained for a day or two to decrease tension on the suture repair.

\section{Results}

The study cohort consisted of 18 severely septic patients (14 men, 4 women) with a mean age of 66 years (range: 50-90 years). At the time of primary laparostomy, the mean APACHE II score was 15 (range: 5-29) and the Mannheim peritonitis index was 34 (range: 28-38). According to the abdominal scoring system by Björck et al. [12] 5 patients are classified as grade 2B, 2 patients as grade 3, and 11 patients as grade 4 , illustrating the severe pathology of this population. Patient characteristics, including the classification according to Björck, are summarized in Table 1. The mean duration with VAC or Bogotá bag treatment, before ABRA application, was 10 days (range: 2-39 days), and the average time from the ABRA application to closure was 15 days (range: 7-30 days). During ABRA treatment, 2 patients died; 1 due to multiple organ failure, 1 due to massive gastric hemorrhage. The average 

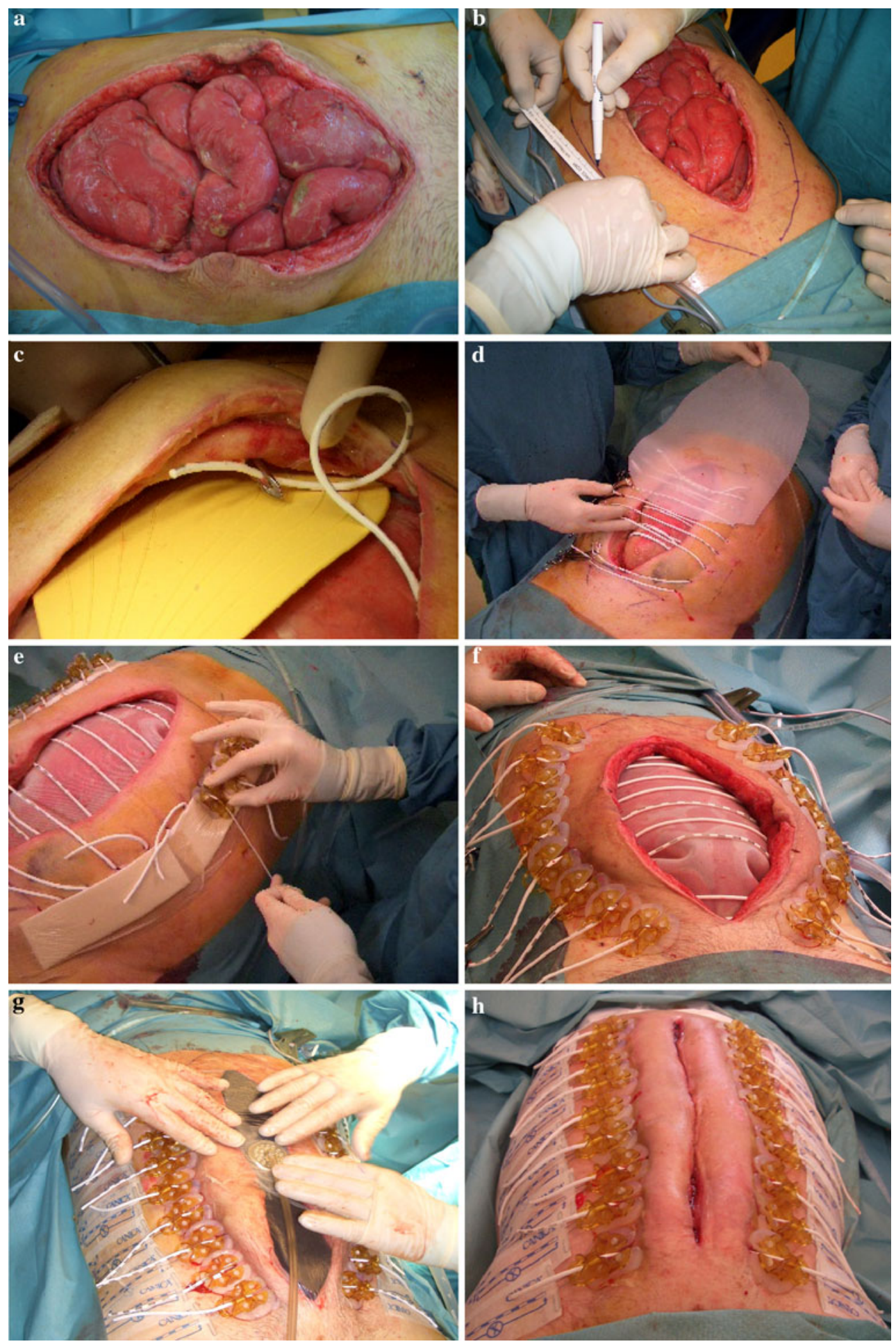

Fig. 1 Procedure of the abdominal re-approximation anchor system. a Abdominal defect during open abdomen treatment, before

sheet and secured by means of the buttons. f ABRA system after surgical application, before applying of Negative Pressure Wound Therapy and button tails. g Negative Pressure Wound Therapy is applied with the ABRA system, on the remaining wound defect. It serves to evacuate abdominal exudate via the perforated silicone sheet. The adhesive button tails are in place and prevent tilting of the buttons. h Image of the abdomen 14 days later, just before removal and definite closure 
length of the total open abdominal treatment for the remaining 16 patients was 25 days (range: $7-48$ days).

The average width of the abdominal defect was $21 \mathrm{~cm}$ (range: 17-27 cm) at ABRA application. The duration of the application procedure ranged from 23 to 90 min (mean: $65 \mathrm{~min}$ ). Typically, in the first few days after application, a local inflammatory response occurred, with a transient expansion of the wound and increased tension. The tension on the elastomers was adjusted accordingly.

We did not encounter any signs of deep infection. However, pressure sores at the anchor sites were encountered in 12 of 18 cases. In 6 cases, the pressure sores were mild; redness around the tunnel of the elastomer was observed from after one week of ABRA treatment up until removal of the system (grade 1 according to Barczak et al. [13]). In another 6 cases, pressure sores of the subcutaneous tissue were observed (grade 2), while muscle and fascia remained unaffected. In 3 of these latter cases deep subcutaneous necrosis (without involving fascia or muscle) occurred. The most severe pressure sore was seen on the contralateral side of a colostomy (Fig. 2a, b; Table 2), implying that too much pressure was exerted on the button at the opposite side of the stoma. Pain developed in 5 patients during ABRA treatment, during the initial phase, when patients were still intubated. This may have been caused by overtensioning the elastomers, and/or induced by reactive edema in the first few days. This pain was severe and had to be treated by administration of morphine and/or ketamine, whereas routinely all patients received nonopioid analgesics.

Primary abdominal closure was accomplished in 14 of the remaining 16 patients $(88 \%)$, by suturing both the fullthickness abdominal layer including fascia, muscle, and skin, without the use of mesh. After closure, there were no
Table 2 Preoperative and postoperative statistics ${ }^{\mathrm{a}}$ in ABRA patients Duration of ABRA operation, min (range) 65 (23-90)

Fascia condition at the time of ABRA application, 6/7/5 undamaged/damaged/severely damaged

ABRA treatment time, days (range)

ABRA related pressure sore (severest grade encountered) ${ }^{\mathrm{b}}$

$\begin{array}{ll}\text { Stage } 1 & 6 / 18 \\ \text { Stage } 2 & 6 / 18 \\ \text { Stage } 3 & 0 / 18 \\ \text { Stage } 4 & 0 / 18 \\ \text { Total period of OA until final closure, days (range) } & 25(7-48)^{*} \\ \text { Mortality } & 2 / 18 \\ \text { Successful delayed primary closure } & 14 / 16^{*} \\ \text { Admission time, days (range) } & 65 \\ & (16-175)^{*} \\ \text { Hernia during follow-up ( } n=16) & 4 / 14^{*} \\ \text { Follow-up time, months (range) } & 23(3-50)^{*}\end{array}$

${ }^{\text {a }}$ Data are presented as mean (range) and are based on the total population $(n=18)$, unless indicated with an asterisk, in which case the number is based on the 16 surviving patients

b Pressure sores were staged according to Barczak et al.; stage 1, skin intact but reddened for greater than $1 \mathrm{~h}$ after relief of pressure; stage 2 , blister or other break in dermis with or without infection; stage 3, subcutaneous destruction into muscle with or without infection and stage 4 , involvement of bone or joint with or without infection

wound infections. In the 2 other patients, an additional component separation technique described by Ramirez et al. [14] was used to close the midline fascia. However, a wound dehiscence occurred in both these patients. One of them suffered from fecal peritonitis in all quadrants after a Hartmann procedure for diverticulitis; the other developed extended peritonitis carcinomatosa and died four months after discharge.
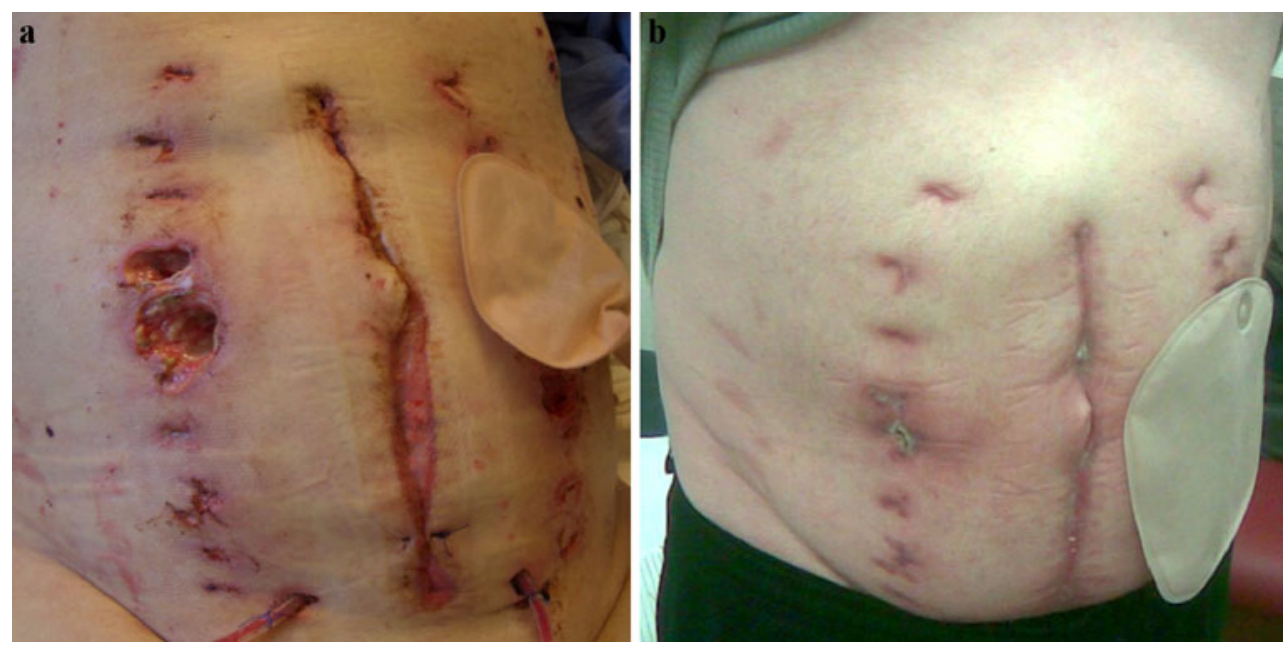

Fig. 2 Pressure sore after use of the abdominal re-approximation anchor system. a Pressure sore anchor button on the contralateral side of the stoma after delayed closure and removal of the ABRA System. b The same patient three months later, during an outpatient clinic follow-up visit 
Furthermore, fistula formation occurred in 3 cases. One patient developed a colocutaneous fistula from a blind distal rectal stump right after sigmoidectomy. Another case developed an enterocutaneous fistula during the VAC abdominal dressing treatment (before application of the ABRA system). The third patient developed an enterocutaneous fistula during ABRA treatment combined with vacuum-assisted closure. These fistulae were treated by means of percutaneous drains. All fistulas closed spontaneously within four months postoperatively.

The average ICU stay was 20 days (range: 7-130 days) and the total hospital admission period was 65 days (range: 16-175 days). In the outpatient clinic, patients were seen every other month during the initial six months and once yearly thereafter; average follow-up is 23 months (range: 3-50 months). Four of the 14 successfully closed patients still developed a midline incisional hernia of around $5 \mathrm{~cm}$ (range: 4-9 cm). Interestingly, two of these patients were scored with extensive fascial damage. Three of the four hernias occurred within six months, and were surgically corrected with a mesh-augmented repair. The fourth patient developed the hernia after 26 months, which remained asymptomatic and was left untreated. The results of ABRA treatment are summarized in Table 2.

\section{Discussion}

In the present study, a novel abdominal wall approximation technique was applied in the infected $\mathrm{OA}$ and delayed closure was achieved in $88 \%$ within 30 days. Previous studies of septic cases with an OA reported delayed primary closure rates between $33 \%$ and $66 \%[15,16]$. In the only multiple case study using the ABRA abdominal approximation system, complete primary closure was achieved in all cases with non-gastrointestinal pathology (such as abdominal compartment syndrome or trauma). However, of the 10 patients with underlying gastrointestinal sepsis, only 4 abdomens could be closed [9]. This is in line with other studies illustrating that the success rate of abdominal closure depends on etiology [8, 17]. Tsuei et al. described both the likelihood and the type of closure (with/ without fascia, mesh, or no closure) to be related to the underlying etiology [8]. Patients with gastrointestinal sepsis or pancreatitis are generally older, less healthy, show higher mortality rates, and are more likely to require a mesh or not reach formal closure at all [8]. In general, it can be stated that sound closure of the infected $\mathrm{OA}$ is more challenging [8, 9, 16-18].

In this respect, it is of great importance to classify the OA status according to Björck et al. [12]. Thereby, patients are not only divided into septic and non-septic causes of OA, but visceral adhesion formation and abdominal wall fixity are also taken into account [12]. Most of our patients had an advanced-stage infected OA (Table 2). In these severe kinds of cases, we have experienced that fascial traction techniques in our hands often fail, due to progressive tearing of the fascia (a complication also described by Gaddnas et al. [4]) during the required prolonged traction. In the present 18 cases, we therefore chose to use the ABRA system, exerting continuous controlled dynamic traction on all layers of the abdominal wall without damaging the fascia during approximation. Our results indicate that the ABRA method is a very suitable alternative for delayed primary closure in these advanced-stage OA cases.

In nonseptic or mildly septic OA cases, we normally use a midline fascial traction method with meshes or a twosided Velcro burr (Wittman patch). This policy is in line with the recent study of Acosta and co-workers, who achieved an $80 \%$ fascial closure rate in OA by vacuumassisted wound treatment and mesh-mediated fascial traction [19]. Their patient population, however, is very different; over $90 \%$ of patients were classified as either Björck grade $1 \mathrm{~A}$ or $1 \mathrm{~B}$, as opposed to only grade $2 \mathrm{~B}, 3$, and 4 patients in our study. One of their conclusions is that the higher the Björck grade, the lower the chance of sound fascial closure. In our opinion, mesh- or Velcro-mediated fascial traction methods are appropriate in the early stages and/or in low-grade OA, whereas the application of ABRA offers additional value in the severely infected high-grade OA. Unfortunately, according to a recently published systematic review and to our best knowledge, no comparative studies for the treatment of the infected OA have been reported [7].

The ABRA system can easily be readjusted at bedside, with a sedative agent and analgesics if necessary, but without anesthesia. This system distributes force over larger surface area instead of converging force on the fascia. However, we realize that this has a significant drawback: the development of pressure sores by transmural traction on the buttons (Fig. 2; Table 2). In most cases, pressure sores were superficial, but grade 2 lesions developed in 6 cases. These patients all had a highly edematous abdominal wall, and elastomer tensioning was performed at its maximum strength (twice the length of the calibration marks). Although all pressure sores eventually healed well, this is a disturbing complication causing pain, discomfort, and unsightly scarring. A more cautious tensioning policy of the elastomers, and additional padding of the buttons with underlying hydrocolloid and gauze dressings may prevent this problem.

Another important factor for successful closure could be the timing of approximation. We applied our approximation system on an average of 12 days after the beginning of OA treatment, compared to 18 days in the previously mentioned study with the ABRA system [9]. In our two 
cases where secondary dehiscence occurred, the system was applied relatively late (after 22 and 39 days), compared to other subjects (all less than 22 days). However, the start of ABRA application in our cases was dictated by clinical judgment, considering the general and hemodynamic condition of the patient, and control of the abdominal sepsis. Nevertheless, it is tempting to speculate that early application at regression of the abdominal sepsis is beneficial for the prevention of fascial retraction, and for reduction of time to closure. This is in line with other studies that acknowledge the importance of preventing fascial retraction [7, 18, 20, 21]

In the presented cases, the etiology of OA was invariably severe peritonitis and not an ACS. Therefore, IAP was not measured before the first acute operation. Throughout ABRA treatment, all patients were hemodynamically stable, and ACS symptoms remained absent. Hence, IAP was not routinely monitored. Since ACS may occur in OA treatment [22], IAP measurements were performed in some patients during VAC abdominal dressing and ABRA treatment in the ICU, but no pathologic values were recorded. However, IAP measurement could be of additional value as a guideline for tensioning of the elastomers or as an indicator for the timing of final closure. Future studies will provide further insight into the usefulness of this parameter.

Regardless of the methods used in OA management, the development of late ventral hernias remains of major concern. In our population of infected OA, 4/16 patients (25\%) still developed a ventral hernia after delayed closure. Interestingly, 2 of these 4 patients were scored with extensive preoperative damage of the fascia. In the previously mentioned study by Reimer et al. with various indications for OA treatment, a ventral hernia developed in $6 / 23$ cases (26\%) [9]. Out of these 6 hernias, 4 occurred in patients with an OA after gastrointestinal sepsis (67\%). The risk of hernia development after delayed primary closure of the OA is likely to be more pronounced in infected abdomens, due to compromised fascial margins, and formation of granulation tissue and adhesions [23]. Both etiology and the condition of the fascia are suggested as playing a role. Therefore, we feel that an effort should be made, especially in advanced stage OA treatment, to spare the fascia from repeated suturing. In contrast to mesh-mediated traction, the traction provided by the ABRA system is dynamic, can continuously be adjusted, and permits both expansion and retraction without damaging the fascia. Even though the septic etiology of OA seems to be a disadvantage with respect to delayed abdominal closure, our results are promising compared to the literature. For future studies, it would be interesting to investigate whether hernias can be prevented by using a synthetic or collagen mesh augmentation at the time of final closure.
In conclusion, our results advocate the abdominal re-approximation system as a useful aid in the management of the infected open abdomen. It would be of value to study this re-approximation system in a prospective, comparative manner. We are currently setting up a national multicenter database for the management of the open abdomen.

Acknowledgments The authors are grateful to Marc Schreinemacher and Ruben Schouten for their critical evaluation of the manuscript.

Conflicts of interest None.

Open Access This article is distributed under the terms of the Creative Commons Attribution Noncommercial License which permits any noncommercial use, distribution, and reproduction in any medium, provided the original author(s) and source are credited.

\section{References}

1. Diaz JJ Jr, Cullinane DC, Dutton WD et al (2010) The management of the open abdomen in trauma and emergency general surgery: part 1-damage control. J Trauma: 68:1425-1438

2. Kaplan M (2004) Managing the open abdomen. Ostomy Wound Manage 50:C2, 1-8, quiz 1p following 8

3. Balogh Z, McKinley BA, Holcomb JB et al (2003) Both primary and secondary abdominal compartment syndrome can be predicted early and are harbingers of multiple organ failure. J Trauma 54:848-859 discussion 859-861

4. Gaddnas F, Saarnio J, Ala-Kokko T et al (2007) Continuous retention suture for the management of open abdomen: a high rate of delayed fascial closure. Scand J Surg: 96:301-307

5. Perez D, Wildi S, Demartines N et al (2007) Prospective evaluation of vacuum-assisted closure in abdominal compartment syndrome and severe abdominal sepsis. J Am Coll Surg: 205: 192-586

6. Nicholas JM, Rix EP, Easley KA et al (2003) Changing patterns in the management of penetrating abdominal trauma: the more things change, the more they stay the same. J Trauma 55: 1095-1108 discussion 1108-1110

7. Boele van Hensbroek P, Wind J, Dijkgraaf MG et al (2009) Temporary closure of the open abdomen: a systematic review on delayed primary fascial closure in patients with an open abdomen. World J Surg 33:199-207

8. Tsuei BJ, Skinner JC, Bernard AC et al (2004) The open peritoneal cavity: etiology correlates with the likelihood of fascial closure. Am Surg 70:652-656

9. Reimer MW, Yelle JD, Reitsma B et al (2008) Management of open abdominal wounds with a dynamic fascial closure system. Can J Surg 51:209-214

10. Urbaniak RM, Khuthaila DK, Khalil AJ et al (2006) Closure of massive abdominal wall defects: a case report using the abdominal reapproximation anchor (ABRA) system. Ann Plast Surg 57:573-577

11. Bosscha K, Reijnders K, Hulstaert PF et al (1997) Prognostic scoring systems to predict outcome in peritonitis and intraabdominal sepsis. Br J Surg 84:1532-1534

12. Björck M, Bruhin A, Cheatham M et al (2009) Classificationimportant step to improve management of patients with an open abdomen. World J Surg 33:1154-1157 
13. Barczak CA, Barnett RI, Childs EJ et al (1997) Fourth national pressure ulcer prevalence survey. Adv Wound Care 10:18-26

14. Ramirez OM, Ruas E, Dellon AL (1990) "Components separation" method for closure of abdominal-wall defects: an anatomic and clinical study. Plast Reconstr Surg 86:519-526

15. Padalino P, Dionigi G, Minoja G et al (2010) Fascia-to-fascia closure with abdominal topical negative pressure for severe abdominal infections: preliminary results in a department of general surgery and intensive care unit. Surg Infect (Larchmt) 11:523-528

16. Wondberg D, Larusson HJ, Metzger U et al (2008) Treatment of the open abdomen with the commercially available vacuumassisted closure system in patients with abdominal sepsis: low primary closure rate. World J Surg 32:2724-2729

17. Schecter WP, Ivatury RR, Rotondo MF et al (2006) Open abdomen after trauma and abdominal sepsis: a strategy for management. J Am Coll Surg 203:390-396

18. Cothren CC, Moore EE, Johnson JL et al (2006) One hundred percent fascial approximation with sequential abdominal closure of the open abdomen. Am J Surg 192:238-242
19. Acosta S, Bjarnason T, Petersson U et al (2011) Multicentre prospective study of fascial closure rate after open abdomen with vacuum and mesh-mediated fascial traction. Br J Surg: 98:735-743

20. Campbell A, Chang M, Fabian T et al (2009) Management of the open abdomen: from initial operation to definitive closure. Am Surg: 75:S1-S22

21. Koss W, Ho HC, Yu M et al (2009) Preventing loss of domain: a management strategy for closure of the "open abdomen" during the initial hospitalization. J Surg Educ 66:89-95

22. Gracias VH, Braslow B, Johnson J et al (2002) Abdominal compartment syndrome in the open abdomen. Arch Surg 137: $1298-1300$

23. Howdieshell TR, Proctor CD, Sternberg E et al (2004) Temporary abdominal closure followed by definitive abdominal wall reconstruction of the open abdomen. Am J Surg 188:301-306 